العدد الثامن عشر(Y I † الجزء الخامس

\title{
L'emprunt dans le discours du cybermarketing : le cas des publipostages
}

Recherche présentée par :

Nirvana Zaki

Sous la direction de :

Mme le professeur

Dr. Nadia Hamdi

Professeur de Linguistique

Faculté des Jeunes Filles

Université Ain Chams
Dr. Riham El Khamissy

Professeur-adjoint de

Linguistique

Faculté de Langues

Trivirmaith A in Thamo

Dr. Samah Nasr

Maitre de conférences

Faculté des Jeunes Filles

Université Ain Chams 


\section{Introduction}

Les mots étrangers incarnent l'interaction perpétuelle entre les langues. Celles-ci, ne pouvant pas développer et maintenir un code linguistique « entièrement autochtone, [et] à l'abri de tout contact avec [...] d'autres langues »(Guilbert, $1975: 89$ ), s'empruntent les unes aux autres afin de satisfaire aux besoins de communication de leurs utilisateurs. Ainsi, tout au long de son histoire, la langue française - à la fois grand fournisseur et récepteur de termes étrangers - a pleinement favorisé l'emprunt linguistique.

L'emprunt représente une des voies ${ }^{1}$ de la néologie reposant non pas sur la création de nouvelles unités mais sur leur insertion dans le système langagier d'une langue donnée même si celles-ci sont déjà produites dans une autre langue. Par conséquent, il constitue une sorte de mine pour le cybermarketing qui œuvre incessamment pour innover le message qu'il véhicule afin de capter l'attention des internautes. Notre corpus constitué de plus de 2500 publipostages ${ }^{2}$ qui nous étaient envoyés suite à notre inscription à plusieurs sites marchands en France, comme H\&M ; Camaïeu; Jules; Pimkie; Brice; Celio ..., pour recevoir leurs nouveautés - en donne un exemple bien évident: les jargons de l'informatique, de la mode ou même du marketing sont envahis surtout par des anglicismes. Ainsi, l'analyse du discours employé en ligne ne peut pas se passer d'étudier le phénomène d'emprunt.

\section{A la recherche d'une définition}

Le terme emprunt est employé à deux sens distincts : 1'acte par lequel on emprunte ainsi que l'élément emprunté. Cette double désignation peut expliquer le fait que ce terme se définit différemment d'un chercheur à un autre. Par exemple, selon Deroy, l'emprunt est « une forme d'expression qu'une communauté linguistique reçoit d'une autre communauté » (Deroy, 1980: 18). Cette définition montre qu'une langue peut comprendre plusieurs communautés linguistiques dont le stock lexical est

\footnotetext{
${ }^{1}$ Les autres voies sont la composition et la dérivation.

${ }^{2}$ Un publipostage est une campagne de marketing direct qui consiste à envoyer une proposition commerciale par email à un ensemble d'individus soigneusement ciblés. (Encyclopédie illustrée de marketing)
} 
distinct. Cependant, elle présente la langue emprunteuse en tant qu'acteur passif qui subit l'acte d'emprunt, ce qui est bien l'inverse.

Deux autres linguistes considèrent l'emprunt comme la solution la plus efficace lorsqu'on ressent le «besoin de dénommer exactement un produit d'origine étrangère ou une réalité typique d'un pays lointain » (Picoche et Marchello-Nizia, 1991 : 338). Or, cette définition désigne en fait un des premiers stades de l'emprunt: le xénisme. Ce dernier peut référer à un produit ou une réalité déjà existants mais qui acquièrent un nouveau sens ou une nouvelle utilisation.

Walter parle d'emprunt « chaque fois qu'une langue prend des mots à sa voisine» (Walter, 1997: 10). En décrivant le vrai processus de ce phénomène, la linguiste refuse la notion même d'emprunter: Walter, pour désigner cet acte, utilise le verbe prendre au lieu d'emprunter puisqu'il ne s'agit pas de la moindre intention de rendre ces mots à la langue prêteuse ${ }^{3}$. Elle limite l'emprunt à un simple mot pris d'une autre langue sans insister sur ses autres formes (phonétique, syntaxique, sémantique, ...). Le choix du terme langue est vague et ne montre pas la complexité de l'emprunt qui peut se produire au sein d'une même langue soit entre ses différents domaines, soit entre ses divers dialectes. La langue française en donne un grand exemple puisqu'elle s'est servie d'éléments empruntés intérieurement aux langues régionales ${ }^{4}$.

Pour Rey-Debove (1998 : 151) l'emprunt n'est qu'un :

« processus par lequel une langue L1, dont le lexique est fini et déterminé dans l'instant $\mathrm{T}$, acquiert un mot $\mathrm{M} 2$ (expression et contenu) qu'elle n'avait pas et qui appartient au lexique d'une langue L2 (également fixe et déterminé dans l'instant $\mathrm{T}$ ) ».

Cette définition met en relief un nouvel élément : la dimension temporelle ayant un grand impact sur la forme définitive d'un terme emprunté. Ainsi, au début de son intégration dans la langue cible (L1), l'emprunt (M2) garde ses caractères étrangers, mais avec le temps il peut acquérir des modifications phonétique, orthographique, sémantique, etc. pour

\footnotetext{
${ }^{3} C f$. Deroy $(1980: 22)$

${ }^{4}$ Parmi ces langues régionales : le basque, l'alsacien, le picard, le normand, le provençal et l'ancien français. $C f$. (Walter, 2005).
} 
s'intégrer davantage au discours de la langue. A un moment donné, il devient complètement assimilé par les utilisateurs de la langue au point qu'il perd toute trace de ses origines et ne sera plus perçu comme intrus. La linguiste considère l'emprunt uniquement comme un processus et le réduit toujours à l'emprunt lexical. La définition qu'elle en donne semble restrictive puisqu'elle n'envisage le mot que comme expression et sens inséparables, excluant ainsi d'autres phénomènes tels que l'emprunt de sens sans l'expression ou l'emprunt de l'expression avec un sens modifié. L'adjectif (fini) insinue la délimitation de la langue (L1) qui recourt à l'emprunt quand son lexique devient achevé et borné face aux nouveaux besoins de communication. Ceci exclut le cas - de plus en plus fréquent depuis la deuxième moitié du $\mathrm{XX}^{\mathrm{ème}}$ siècle - où la langue offre bien des équivalents mais ses utilisateurs préfèrent opter pour des emprunts.

Dubois (2002 : 177), quant à lui, propose une définition qui nous paraît plus précise et plus adéquate :

«Il y a emprunt linguistique quand un parler A utilise et finit par intégrer une unité ou un trait linguistique qui existait précédemment dans un parler $\mathrm{B}$ et que $\mathrm{A}$ ne possédait pas; l'unité ou le trait emprunté sont eux-mêmes appelés emprunts. L'emprunt est le phénomène sociolinguistique le plus important [...]. Il est nécessairement lié au prestige dont jouit une langue ».

Cette définition envisage l'emprunt tant comme processus que comme élément emprunté. Elle ne réduit pas ce dernier au type lexical, par contre, elle laisse la voie ouverte à toute unité linguistique (phonème, morphème, sémème, etc.). Elle est donc englobante. En outre, elle n'exclut ni le cas où équivalents et emprunts cohabitent au sein de la même langue ; ni le cas des allers-retours entre les langues. Pour parler de la lexicalisation de l'emprunt, le recours au verbe utiliser complété par le verbe intégrer est très précis car le premier décrit exactement l'action d'emprunter tandis que l'autre indique son stade final. Conscient qu'une seule langue peut contenir plusieurs idiomes, Dubois a minutieusement employé le terme parler afin d'inclure l'emprunt interne. La particularité de cette définition réside dans l'introduction de la dimension sociolinguistique qui est incontestablement le chef d'orchestre en matière d'emprunt. Au-delà du simple besoin de dénomination, le vrai motif 
d'emprunter se justifie par le prestige de la langue source qui le gagne à cause de son poids économique, son pouvoir politique, son progrès technologique et scientifique, sa culture imposante ou sa présence massive.

Un phénomène ayant tant de définitions ne facilitera pas la tâche des linguistes pour en dresser une typologie. Les catégories de l'emprunt s'avèrent être multiples parce que les chercheurs le classent selon plusieurs niveaux (la source, les motifs, l'intégration, la nature linguistique) qui, à leur tour, se divisent en différents sous-types. La complexité de la tâche réside dans le fait qu'une seule unité empruntée accepte d'être répertoriée sous chacun de ces niveaux. Pour en sortir, nous avons opté pour une classification inspirée de certains travaux de Sarah Leroy (2010). Notre intérêt est de rendre compte de la richesse de l'emprunt qui - dans notre corpus - se manifeste sur trois plans différents : emprunt inter-langues, emprunt inter-discours et emprunt inter-sociétés. Nous étudierons plutôt les emprunts qui ne sont pas encore lexicographiés ${ }^{5}$ puisque nous cherchons l'apport néologique qui caractérise le discours du cybermarketing, tout en braquant la lumière - le cas échéant - sur certains emprunts déjà existants.

Pour le présent article, nous nous limitons au premier plan seulement, celui de l'emprunt inter-langues où nous essaierons d'en dresser une typologie en fonction des exemples recensés dans notre corpus. Nous présenterons ensuite certains emprunts pour voir à quel point ils sont intégrés au sein de la langue française et s'ils sont nécessaires ou non. Nous nous efforcerons, en fin, de répondre à une question incessante : le recours à l'emprunt dans le discours en ligne traduit-il la créativité des annonceurs ou - au contraire - leur paresse ?

\section{L'emprunt inter-langues}

Afin d'identifier un emprunt, il est indispensable d'en poursuivre les origines. Quoique parfois bien dissimulées, elles décèlent la présence de contacts ininterrompus entre la langue française et toute une variété d'autres langues. Si l'internaute consultant sa boîte aux lettres peut

\footnotetext{
${ }^{5}$ D'après Sarah Leroy, la lexicographisation c'est la présence d'un terme dans les dictionnaires de langue. $C f$. (Leroy, 2006 : 66).
} 
rencontrer - de temps en temps - quelques mots espagnol, italien ou même arabe, il est le plus souvent inondé par des anglicismes. Examinons les exemples suivants :

(1). "Pour être au cœur de la tendance, Kiabi a sélectionné les must-have à shopper sans modération!'” (Kiabi, 14/10/15)

(2). 'Immanquable! Les deals de l'extrême : jusqu'à 90\% d'économie !' (Pimkie, 14/10/15)

(3). "Les plus beaux perfectos ${ }^{6}$ sont arrivés! Retrouvez notre sélection en magasin ou sur promod.fr" (Promod, $14 / 10 / 15)$

(4). "Essential Prices: des pièces clé à des prix que vous adorerez" (Mango, 14/10/15)

(5). "Fever Nights! du $18 \mathrm{H}$ à minuit $-30 \%$ dès $45 €$ d'achat !" (Jules, 14/10/15)

De prime abord, évidente est l'empreinte de l'anglicisme qui apparaît dans quatre sur cinq publipostages envoyés tous le même jour. L'emprunt à l'espagnol dans le terme «perfecto » (ex. 3), dont la graphie est très remarquable, est employé dans la nomenclature d'un produit de mode ; ce qui peut - dans une certaine mesure - être toléré, alors que les emprunts à l'anglais dans les autres exemples relèvent de la langue commune «must, deals, prices, fever, etc. » et n'ont donc aucune raison de s'introduire dans la langue française qui possède certainement des équivalents. Leur abondance dans les publipostages mérite d'être étudiée.

\subsection{Le grand fournisseur}

Partout dans notre corpus, l'anglais est employé tant pour une nécessité que pour un luxe. Les nouvelles technologies de l'information et de communications (NTIC) parlent en anglais. La mode - même si elle n'est pas l'apanage des américains - choisit des noms anglais pour ses produits et ses coupes. Les termes du français général se trouvent substitués par des anglicismes. En effet, les concepteurs du texte publicitaire affichent un engouement pour la langue anglaise dans le but de pimenter le message avec une touche étrangère, à un tel point que l'anglais devient la source la plus importante d'emprunts.

${ }^{6}$ Un blouson en cuir de cheval qui possède une coupe spécifique avec fermeture croisée et zippée, des poignets zippés et une ceinture dans le même cuir que le blouson. 
Certes, la couleur anglaise qui teinte le discours du cybermarketing se manifeste davantage dans le coté morphologique mais elle touche également d'autres cotés (phonétique, syntaxe, etc.). Ainsi est-il important d'analyser ces nouveaux venus selon leurs natures linguistiques.

\subsection{Typologie des emprunts}

L'analyse des emprunts doit être établie conformément à la nature de l'élément emprunté parce qu'elle est considérée comme le cadre le plus petit et le plus minutieux dans la démarche de l'identification à l'opposé des autres angles d'analyse tels que les motifs, l'intégration ou la nécessité qui abordent ce phénomène d'une manière plus générale. La typologie basée sur la nature linguistique renferme six grands types (lexical, syntaxique, phonétique, sémantique, calque et faux emprunt) dont quelques-uns sont divisés en sous-types.

\subsubsection{L'emprunt lexical}

C'est le type le plus fréquent et le plus explicite. Il traite essentiellement des lexèmes pris à une autre langue et comprend trois sous-types :

\subsubsection{Emprunt intégral (forme et sens)}

Une lecture rapide dans notre corpus nous révèle qu'il regorge d'emprunts de sous-type intégral. Nous en avons relevé certains exemples ${ }^{7}$ dont :

(6). “Jules est ouvert le dimanche, yes !'” (Jules, 20/12/14)

(7). "Happy xmas avec un look chic et cool ou Happy new year avec une allure chic et choc ?' (Camaïeu, 05/12/13)

(8). "Le gilet long coupe loose ... $\mathrm{Du} 46$ au 60 : NEW !' (Kiabi, 27/09/14)

(9). "La doudoune super Light est de retour" (Camaïeu, $15 / 10 / 16)$

(10). "La doudoune bi-matière [...]. A porter avec un chino marron et des sneakers pour une touche casual" (Celio, 03/11/15)

\footnotetext{
${ }^{7}$ Les termes soulignés - dans ces exemples - sont des emprunts déjà lexicographiés, alors que les termes en italique ne le sont pas encore.
} 
(11). “Bye Bye l'été ! Welcome les prix légers!” (Jules, 23/09/14)

(12). "Looks rock, sexy et sporty pour cet automne" (Kiabi, 14/09/15)

(13). "CC'est $\underline{\text { Hot, }}$ c'est Now : Les bottines must-have de l'hiver" (H\&M, 21/10/13)

(14). “Les pulls oversize se mixeront élégamment aux jupes étroites"' (Pimkie, 15/10/15)

A tort ou à raison, les annonceurs du cybermarketing ont emprunté ces termes avec leurs formes typographiques, leurs sens et leurs concepts. Il parait que le nombre des anglicismes déjà figurants dans les dictionnaires - bien qu'il ne soit pas du tout négligeable - leur semble insuffisant ; ils augmentent le stock lexical par un nouvel arrivage. En effet, ces emprunts ne font que traduire la fascination par tout ce qui est étiqueté «américain ».

2.2.1.2. Emprunt hybride (forme partielle et sens).

Il représente le cas de dérivation où la langue ajoute des morphèmes à un radical étranger soit pour pouvoir accueillir l'emprunt dans son système linguistique, soit pour en créer une série de dérivés ${ }^{8}$.

(15). "Si vous ne visualisez pas correctement ce message, cliquez-ici”' (Jules, 12/12/13)

(16). "Un complet gris chiné mixé à un chino cuivré pour une allure de gentleman" (Brice, 05/11/14)

(17). "Cet automne, les tons se mixeront sur une large palette de couleurs chaudes"' (Pimkie, 22/09/16)

(18). "Mixez et Matchez vos maillots de bain pour cet été", (Kiabi, 18/06/16)

(19). "Légendez ce look avec un commentaire. Le commentaire le plus liké remportera une carte cadeau de 20€' (Jules, 30/04/16)

Dans ces exemples, les bases verbales sont des radicaux empruntés à l'anglais avec la terminaison du français soit de l'impératif (ex. 15 et 18), du participe passé (ex. 16 et 19) et du futur simple (ex. 17). Certains de

${ }^{8} C f$. (Loubier, $\left.2011: 14\right)$. 
ces anglicismes sont liés au domaine de l'Internet où les Etats-Unis sont des pionniers.

\subsubsection{Emprunt partiel (forme seule)}

Pour certains linguistes, le sous-type partiel qui porte sur la forme graphique du terme, prête à confusion, notamment lorsqu'il s'agit d'un anglicisme. Quelques-uns le prennent pour un faux emprunt. Loubier (2011 : 14) déclare que «parfois, le faux emprunt résulte d'un emprunt limité à la forme. Il donne lieu à des sens différents selon la langue [cible] ». Rey-Debove (1998: 171) trouve que «ces emprunts purement formels sont qualifiés de « faux anglicismes » ou «pseudo-anglicismes ».

Cette confusion, en fait, ne nous paraît pas justifiée puisque la définition fournie par Loubier lui-même est assez nette. Le faux emprunt est composé - de la part des utilisateurs de la langue cible - de deux éléments étrangers mais sa forme finale n'existe pas dans la langue source et n'y a aucun sens. Alors que l'emprunt partiel consiste en un terme qui existe pleinement dans la langue source et qui acquiert un sens différent dans la langue cible. Examinons ces exemples :

(20). "Profitez-en pour faire le plein de slip" (Jules, 08/02/15)

(21). "Optez pour un look jean baskets actualisé" (H\&M, $02 / 03 / 16)$

(22). “La sélection fête pour vous ! On opte sans hésiter pour la veste du smoking et la chemise slim-fit" (Celio, 17/05/16)

Nous avons affaire ici à trois emprunts partiels. Dans l'exemple (20), le terme « slip » est un emprunt à l'anglais utilisé pour désigner - d'après les dictionnaires Oxford Living $^{9}$ et Cambridge ${ }^{10}$ - un vêtement ample et flottant porté par les femmes sous leurs vêtements (robe, jupe, etc.). Le Dictionnaire Larousse (anglais/français) lui donne comme synonymes : «combinaison, fond de robe, jupon ${ }^{11}$. Une fois entré dans la langue française, le terme «slip » se trouve défini dans le même dictionnaire version (français/français) - comme « une culotte moulante à taille basse

\footnotetext{
${ }^{9}$ Dans Oxford Living, slip signifie « A loose-fitting garment, typically a short petticoat. » Synonyms : « underskirt, petticoat», in https://en.oxforddictionaries.com/definition/slip (consulté le 28/10/16)

${ }^{10}$ Dans Cambridge, slip signifie " A kind of undergarment worn under a dress, a petticoat. ", in http://dictionary.cambridge.org/dictionary/english-french/slip_2 (consulté le 28/10/16)

${ }_{11}$ Dans Larousse en ligne, http://www.larousse.fr/dictionnaires/anglais-francais/slip/612507\#642457 (consulté le 28/10/16)
} 
servant de sous-vêtement ${ }^{12}$ sans précision de sexe. Sachons que le même signifié, en anglais, a pour signifiant les termes « underpants » (américain et britannique) ou «shorts » (américain) pour homme ainsi que «briefs », «panties » (américain) ou « knickers » (britannique) pour femme. C'est ainsi que «slip » s'avère être un emprunt partiel dont la forme est anglaise mais le sens est français.

Il en est de même pour l'exemple (21): «Baskets » est un anglicisme désignant en français des chaussures sportives ou pas formelles. En anglais, ce terme signifie un panier, et moins souvent le filet constituant le but du basketball. Pour parler de chaussures pas formelles, l'anglais ${ }^{13}$ utilise : sneakers (américain) ou trainers (britannique).

Le troisième emprunt mérite une attention particulière. Il est erronément envisagé en tant que faux-emprunt alors que le terme existe effectivement en anglais mais avec un sens différent. Le nom «smoking » renvoie selon Larousse - à une tenue de soirée masculine dont le veston est à revers de satin. Or, cette signification est valide partout sauf au pays de la langue source où «smoking» ne désigne que l'action de fumer (le participe présent du verbe to smoke et - par glissement de catégorie l'adjectif). Le costume est appelé «taxedo» chez les américains et «dinner jacket » chez les anglais ${ }^{14}$.

\subsubsection{L'emprunt syntaxique}

On parle d'emprunt syntaxique chaque fois une langue utilise de nouvelles structures qui ne sont pas conformes à sa phraséologie ${ }^{15}$. A l'instar de la langue anglaise, la syntaxe française tantôt change la place de l'adjectif, tantôt marque l'appartenance à l'aide de ('s) comme dans :

(23). “Fantastiques offres pour les fêtes! Vite!" (ASOS, 14/11/14)

(24). “Le look du moment ... C'est le bleu, blanc look” (Jules, 04/11/13)

(25). '“Les Jules's days, c'est parti'” (Jules, 18/09/13)

\subsubsection{L'emprunt phonétique}

\footnotetext{
${ }^{12} \mathrm{Ibid}$, http://www.larousse.fr/dictionnaires/francais/slip/73052?q=slip\#72226 (consulté le 28/10/16)

${ }^{13} \mathrm{Cf}$. http://dictionary.cambridge.org/dictionary/english/trainer (consulté le 28/10/16)

${ }_{15}^{14} C f$. http://dictionary.cambridge.org/dictionary/english/tuxedo?a=british (consulté le 28/10/16)

${ }^{15} C f$. Loubier (2011: 15$)$
} 
C'est le cas où la langue adopte une prononciation étrangère ${ }^{16}$. Les phonèmes anglais se font entendre parmi les utilisateurs du français :

(26). “-40\% \& $-30 \%$ sur notre sélection et une surprise sur la $\underline{e}$ boutique !" (Comptoir des Cotonniers, 15/03/16)

(27). "Vous pouvez essayer les articles avant de les acheter grâce à l'é-réservation et en plus, c'est gratuit !' (Tape à l'œil, 15/08/16)

(28). 'Les Jours Camaïeu : encore plus de produits jusqu'à $50 \%$ ! Les sneakers unis à 27€”' (Camaïeu, 28/08/25)

(29). "Découvrez et succombez pour notre produit de la semaine: Le Jegging prêt pour toutes les saisons!" (Promod, 12/06/15)

(30). 'Ce sera aussi l'occasion de sortir nos pièces les plus mode : manteaux oversize, capes, longs gilets [...]" (Blancheporte, 07/10/14)

Dans ces exemples, la prononciation anglaise est adoptée : [i] dans (êcarte et e-réservation); $[\mathrm{z}]$ dans (sneakers) ; [j] dans oversize et $\left[\mathrm{d}_{\mathrm{z}}\right]$ dans (jegging).

\subsubsection{Le calque}

Il s'effectue à travers deux procédés différents : la traduction proprement dite et le vrai calque étant "aussi une traduction, mais une traduction littérale, c'est-à-dire conforme aux structures de la langue d'origine"' (Treps, $2003: 245$ ).

(31). “J 2 des soldes ! Préparez vos soldes en 3 clics” (Celio, $03 / 07 / 15)$

(32). "Retardataires ? Pas de panique! Pensez à l'e-carte" (Jules, 20/12/13)

Ces unités d'apparence française sont calquées respectivement sur des unités anglaises (Day 2) au lieu de ( $2^{\text {ème }}$ jour) et (e-card) au lieu de (carte électronique).

(33). 'II suffira [...] de l'activer en ligne”' (Pimkie, 24/05/14)

Ici, l'unité est traduite de l'anglais (on line).

${ }^{16} C f$. Loubier (2011: 16) 


\subsubsection{Le faux emprunt}

C'est un terme "qui a l'apparence d'un emprunt intégral et qui est constitué d'éléments formels empruntés, mais sans qu'aucune unité lexicale (forme et sens) ne soit attestée dans la langue prêteuse » (Loubier, 2011 : 14). Ce type est absent dans notre corpus. Cependant, l'intitulé « faux emprunt » peut - à notre sens - être appliqué aux allersretours. Ces derniers consistent en un élément qu'une langue prête à une autre et le réemprunte plus tard avec, sans doute, quelques modifications. Le domaine de la mode en donne deux exemples très fréquents dans les publipostages.

(34). "Déclinée sur le thème du voyage, la collection Studio H\&M S/S 2016 mixe denim, pièces artisanales et imprimés éclatants." (H\&M, 03/03/16)

(35). "Enfin: votre nouveau jean fétiche! Trouvez le votre dans notre vaste collection !'” (H\&M, 29/02/16)

Le $\mathrm{TLFi}^{17}$ mentionne l'étymologie française des termes «denim» et «jean». Vers la fin du XIX ${ }^{\text {ème }}$ siècle, les pantalons jeans ou conformément à leur première appellation - les «blue-jeans » étaient fabriqués à Nîmes, une commune du sud-est de la France. Ce tissu composé d'un mélange de laine et de coton - était réputé surtout par sa résistance et appelé donc «tissu de Nîmes », ce qui s'est transformé avec le temps en denim [de Nîmes $\rightarrow$ denim]. La couleur bleue utilisée dans la teinture du tissu de Nîmes venait d'une autre commune française, Gênes. Pour distinguer la couleur bleue de cette étoffe (tournant vers l'indigo), on l'appelait bleu de Gênes, ce qui a abouti enfin à blue-jean. Avec l'essor de l'industrie des jeans aux Etats-Unis, les deux appellations « de Nîmes » et «bleu de Gênes » ont regagné le français sous une fausse nationalité américaine : denim et blue-jean. C'est en fonction de leur apparence faussement empruntée, que nous annexons les allers-retours à ce type d'emprunt.

Le concept même des allers-retours nous incite à étudier de plus près le trajet que suit un terme donné de la langue source jusqu'à son intégration dans la langue cible.

\footnotetext{
${ }^{17}$ Dictionnaire le Trésor de la Langue Française informatisé.
} 


\section{L'intégration des emprunts}

Le transfert de l'emprunt d'une langue vers une autre et sa transplantation dans celle-ci se produit selon un long processus composé de trois stades dont les définitions ne trouvent pas un accord unanime de la part des linguistes. Le nouvel élément emprunté commence tout d'abord par être un xénisme considéré comme un « terme étranger [qui] est introduit dans le corps d'une phrase [...] en référence à un signifié propre à la langue étrangère » (Guilbert, 1975 : 92). Il est employé surtout par les médias et la littérature dans le but de créer une ambiance exotique et d'évoquer la couleur locale du sujet mentionné.

S'il survit au sein de la langue cible et s'intègre de plus en plus à son lexique, il devient un pérégrinisme qui «renvoie encore à la réalité étrangère, mais la connaissance de son sens est supposée partagée par l'interlocuteur» (Dubois, 2002: 512). Il est à signaler que le pérégrinisme peut subir quelques légères adaptations (ajouter la marque du pluriel ou transformer un phonème) afin de mieux s'intégrer dans la langue source.

Avec le temps et l'emploi fréquent, il atteint son stade ultime et finit par être un emprunt tout à fait intégré dans la communauté linguistique, répandu dans son vocabulaire et assimilé par ses membres. Cette assimilation «s'effectue à tous les niveaux de la langue, celui de la prononciation, de l'orthographe, de la morphologie, du lexique et de la syntaxe » (Humbley, $1974: 66)$.

Il est clair que la distinction entre ces trois stades dépend étroitement de l'usage discursif du nouveau venu : plus son signifiant est connu par la masse parlante, plus son intégration est avancée. Ceci dit, nous tentons d'observer dans quels stades peuvent se fixer les emprunts recensés dans notre corpus.

Dans un publipostage ${ }^{18}$ envoyé le 10 octobre 2016, apparaît l'emprunt suivant qui incarne le passage du xénisme au pérégrinisme :

(36). “'Où trouver l'offre Burkini au meilleur prix ? Dans le magasin Prêt-à-Porter Cdiscount bien sûr !’' (Cdiscount, 10/10/16)

\footnotetext{
${ }^{18}$ Ce publipostage nous a été envoyé suite à notre question posée à travers notre compte sur le site du magasin et demandant les dernières offres de Burkini.
} 
Suite à la polémique ${ }^{19}$ estivale suscitée en France concernant le maillot de bain porté par les femmes musulmanes sur les plages de l'Hexagone, le terme «burkini » se voit massivement employé dans la presse française. Au début de l'affaire qui a commencé en août 2016, les journaux - dont Le Monde et Libération - citaient toujours ledit terme avec sa définition présentée d'une manière nette au sein même de l'article ${ }^{20}$. Alors, il était jusqu'à ce moment-là considéré comme un xénisme qui "peu ou pas intégré à la langue cible, reste un mot étranger, mentionné avec référence au code [culturel] d'origine, et [représentant une des] réalités étrangères » à la société française (Leroy, 2006: 67). Peu à peu, la définition commençait à apparaître en marge de l'article jusqu'à sa disparition complète $^{21}$ vers les débuts du mois de septembre 2016. Dès lors, «burkini » pouvait se classer parmi les pérégrinismes « qui, tout en renvoyant à une réalité [étrangère], sont suffisamment intégrés pour pouvoir être employés sans explication particulière » (Leroy, 2006 : 67).

Quant aux autres emprunts faisant partie de notre corpus, ils sont - pour la majorité - des anglicismes; ce qui constitue un cas délicat dans le processus de l'intégration. Etant une langue universelle parlée et étudiée dans les quatre coins du monde, l'anglais ne possède pas de réalités qui lui sont propres : ses inventions, ses termes, ses expressions et tous les domaines de la vie qui s'y attachent sont rapidement répandus sur le plan mondial et intégrés partout. Il en résulte que ses produits linguistiques ne renvoient pas à une culture donnée et - par conséquent - gagnent une chance de passer directement au troisième stade, celui de l'emprunt. S'ils ne sont pas encore lexicalisés, ils sont facilement compris de la part de la masse parlante et s'installent aisément dans les langues cibles. L'exemple suivant présente un anglicisme largement employé dans l'univers de la mode :

\footnotetext{
${ }^{19}$ Une association de femmes musulmanes

${ }^{20}$ Exemples d'articles mentionnant « burkini » avec sa définition :

- http://www.lemonde.fr/les-decodeurs/article/2016/08/05/polemique-sur-le-burkini-a-marseillece-qu-il-faut-savoir 4979016 4355770.html

- $\quad$ http://www.liberation.fr/france/2016/08/12/a-cannes-le-maire-prend-la-pose-sur-leburkini_1471885

${ }^{21}$ Exemples d'articles mentionnant « burkini » sans définition :

- http://www.lemonde.fr/port-du-voile/article/2016/09/06/le-tribunal-administratif-de-bastiavalide-l-arrete-anti-burkini-de-sisco_4993489_4987696.html

- $\quad$ http://www.liberation.fr/debats/2016/09/07/burkini-la-france-contre-le-reste-du-monde_1488687
} 
(37). “Nouveau lookbook de novembre 2015, à ne pas rater !" (Mango, 27/10/15)

Bien que le «lookbook» ne figure pas dans les dictionnaires de langue, les cyberclients peuvent - sans effort - en déchiffrer le sens : un catalogue (book) de photos exposant la nouvelle collection (look) de chaque marque. Se pose ici une incessante question: si la langue française possède déjà un signifié autochtone pour cette réalité, faire appel à un tel emprunt serait-il vraiment nécessaire ?

\subsection{L'emprunt entre nécessité et luxe}

Les motifs incitant à chercher un terme ailleurs peuvent être légalement justifiés par une pénurie lexicale. Nous parlons dans ce cas de l'emprunt de nécessité, appelé également «emprunt dénotatif»par Guilbert, et représentant « les désignations de produits, de concepts qui ont été créés dans un pays étranger» (Guilbert, 1975: 91) et ne trouvant pas d'équivalent dans la langue source. Etudions ces exemples :

(38). "Pour fêter ce match et soutenir notre équipe, Celio vous offre $-10 \%$ sur tous les articles (SOLDES compris)!" (Celio, 01/06/16)

(39). "Ouverte jour et nuit, la boutique pyjamas fille vous réserve des pyjamas et chemises de nuit de rêve ... à concrétiser en 1 clic'" (Tape à l'œil, 12/07/15)

(40). 'Succombez à toutes vos envies : sport, détente, mode, à talons ... tous les styles de chaussures sont sur Kiabi.com", (Kiabi, 23/10/16)

Bien que ces emprunts soient depuis très longtemps intégrés dans la langue française, cette dernière n'a pas pu - jusqu'à présent - offrir des remplaçants nationaux. Il en est de même pour certains anglicismes relativement plus récents :

(41). “Le It du moment: la chemise fluide!' (Promod, 29/07/16)

Pour parler d'un produit ou d'un look représentant la dernière tendance de la mode et généralement porté par une star ou pour braquer la lumière sur un produit qui est réintroduit avec une star ou un designer célèbre, les 
concepteurs de publipostages sont contraints de recourir au terme anglais «it ».

L'évolution technologique notamment dans le domaine de l'informatique et l'expansion des réseaux sociaux imposent l'emprunt de nouveaux termes purement techniques. L'anglais, étant la langue prédominante de ces champs, se trouve dans des nomenclatures telles que :

(42). "Partagez vos looks Jules avec le hashtag ${ }^{22}$ \# ILPARAIT" (Jules, 17/10/14)

(43). "On se trouve sur Instagram! Postez une selfie $e^{23}$ avec un look Jules"' (Jules, 14/11/15)

Quelques courants de mode vestimentaire n'ont pas changé leurs désignations pour correspondre à la langue accueillante comme :

(44). "Osez le pull urbain color block ${ }^{24, " ~(C a m a i ̈ e u, ~ 08 / 05 / 14) ~}$

(45). "Encore plus d'articles de saison en soldes: la veste

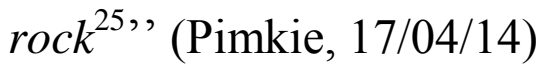

(46). "Le blouson: un look pop $^{26}$ incontournable et ultra pratique pour la mi-saison"' (Mango, 22/09/13)

Certaines coupes de pantalon gardent leurs appellations anglaises faute d'alternatives françaises comme :

(47). "Quel jean est fait pour vous? L'indispensable slim ou l'incontournable bootcut? Faites votre choix [...] dès 19,95€ !' (Camaïeu, 13/03/14)

(48). 'Jusqu'à $-80 \%$ : Legging, jupes, vestes, robes ... Faitesvous plaisir !' (Blancheporte, 24/07/15)

(49). "Ne loupez rien de la nouvelle collection $[\ldots]: 10 €$ le skinny (disponible en 10 coloris)" (Kiabi, 11/09/16).

A côté de ces emprunts de nécessité, nous avons constaté un autre type qui nous semble «quasi nécessaire » où le nouveau venu - malgré la

22Mot clé servant à référer les micro-messages sur les réseaux sociaux.

23 Autoportrait fait à l'aide d'un appareil photo ou photophone.

${ }^{24}$ Tendance de la mode renvoyant à la combinaison de couleurs unies très vives qui ne se portent généralement pas ensemble pour produire un choc à l'œil comme par exemple, porter une chemise bleue sur un pantalon rouge avec des chaussures vertes et un sac jaune.

${ }^{25}$ Un style de vêtement décontracté et généralement en cuir.

${ }^{26}$ Un style de vêtement très décontracté et confortable. 
présence d'un équivalent français - ajoute une différente connotation ou une précision absente du terme autochtone. Comparons ces exemples :

(50). "La fête des mères approche ! découvrez notre sélection : les sandales à talons carrés à 17€"' (Promod, 17/04/16)

(51). "Oh les basiques à petits prix : les tongs ${ }^{27}$ unies à partir de $2 €$ !'” (Kiabi, 23/05/15)

(52). “Imprimé fruit, fleuri ... et le tout à moins de $10 €$ ! Ça vous tente?: Le soutien-gorge femme /10€. La culotte /6€', (H\&M, 01/08/15)

(53). 'Dernier jour, 3 pour le prix de 2 : Lot de 3 strings dentelle à 7,70€', (Pimkie, 09/02/14)

(54). "Faites le plein de confortables sous-vêtements grâce à notre super promo : tangas à $5^{€ 90,, ~(H \& M, ~ 29 / 07 / 15) ~}$

Dans les exemples (50 et 51), le terme «sandales» a gagné plus de spécification sur la forme grâce à l'emprunt «tongs ». Il en est de même pour les exemples (52 à 54) : les lexèmes «string» et «tanga ${ }^{28}$ ont précisé davantage la coupe de la « culotte».

D'autres emprunts peuvent être - à notre sens - qualifiés de «quasi nécessaire » lorsqu'ils sont employés pour éviter soit un long syntagme soit une équivoque :

(55). "Créez votre slogan. Le slogan qui reçoit le plus de likes remportera une carte cadeau de 20€' (Brice, 12/11/15)

(56). "Ça réchauffe de rester en contact ... Je like Jules ! Je follow Jules!'” (Jules, 14/01/14)

Devant l'équivalent français qui se forge d'un nom + une phrase «la mention "j'aime" », le substantif « likes » (ex. 55) parait plus bref et donc plus favorable. Si le verbe «suivre » avait été employé au lieu de «follow », l'internaute l'aurait pris pour le verbe « être »: je suis Jules. Quant au terme « like» (ex. 56), remplaçant le verbe « aimer », il s'avère vain et fait alors partie des emprunts de luxe.

\footnotetext{
${ }^{27}$ Tong : sorte de sandales qui laisse montrer les orteils des pieds nus tout en séparant le gros orteil des autres.

${ }^{28}$ String et Tanga : deux coupes de sous vêtement.
} 
L'emprunt de luxe ou « connotatif » représente le cas où les utilisateurs d'une langue peuvent emprunter un terme « logiquement inutile [...] qui a été pris alors qu'une désignation existait ou était possible dans la langue emprunteuse » (Deroy, $1980: 170-171)$. A titre d'exemples, nous citons :

(57). “-50\% sur les shoes d'hiver! Profitez-en vite” (H\&M, $12 / 02 / 14)$

(58). 'La journée va être longue du réveil à l'after work. Un petit côté preppy pour cette tenue urbaine, où la veste tweed se marie à un jean." (Jules, 01/10/13)

(59). "Le pantalon straight, un incontournable du dressing féminin" (Camaïeu, 15/03/14)

(60). "Dernière ligne droite avant le week-end, porte ouverte au friday wear! Optez pour un jean baskets réactualisé." (Celio, 27/02/16)

(61). "Du vendredi 04 au jeudi 17 décembre inclus, venez profiter des CRAZY Days uniquement sur Kiabi.com", (Kiabi, 09/12/15)

(62). "Jules vous souhaite un Happy Xmas" (Jules, 24/12/13)

(63). "Un gilet loose comme on les aime, tendance et coloré !' (Promod, 26/08/15)

(64). 'La méduse ${ }^{29}$ has been en force! Soyez toujours élégante avec la fameuse méduse en plastique en toutes couleurs" (Pimkie, 09/06/14)

(65). “Dernière démarque : Jusqu'à -90\%! Go !” (ASOS, 31/07/15)

(66). 'Hello SUN ! Votre dressing de plage, par ici !' (Kiabi, 07/06/15)

(67). "Cet hiver so british: le légendaire trench est l'arme de séduction idéale !"” (Brice, 11/11/14)

(68). “Xmas GIFT: -10€ pour l'achat de 2 articles" (Petit Bateau, 01/12/15)

Dans la concurrence des mots, ces emprunts de luxe ont pris le dessus sur leurs équivalents français bien existants. Or, aucune raison ne justifie la présence de ces unités anglaises surtout lorsqu'ils coexistent avec des équivalents français dans le même texte.

${ }^{29}$ Sandales en plastique souple et transparent formées de plusieurs lanières et couvrant les orteils. 
(69). “'Osez les imprimés graphiques black and white! En robe, en top ou en chemise, le noir et blanc sont des pièces intemporelles de votre dressing" (H\&M, 11/01/16)

(70). “Un bon dressing démarre par de bons Basics ! [...] Voir Les Basiques Femmes.” (Kiabi, 31/07/15)

Si la langue a ses raisons pour tolérer les emprunts de nécessité, les emprunts de luxe, en raison de leur inefficacité et leur présence massive non seulement dans les publipostages mais aussi dans le langage du cybermarketing, mettent en question l'essence même du phénomène d'emprunt.

\section{L'emprunt : une créativité ?}

Controversé pendant des décennies, le phénomène de l'emprunt ne cesse d'engager des débats entre les linguistes. Ces débats ont atteint leur apogée pendant le $X^{\text {ème }}$ siècle avec la montée extrême de l'emprunt à l'anglais. La situation est devenue alarmante vers les années cinquante à cause de l'emprunt massif à l'américain. Les puristes y voient une menace non uniquement linguistique mais aussi socio-politique. Par tous les moyens, ils affichent leur indignation de l'invasion des termes étrangers notamment d'outre-Atlantique et provoquent le sentiment nationaliste pour résister à ces « nouveaux colonisateurs ».

Le Parlez-vous franglais? d'Etiemble consiste en une véritable alerte contre le «prestige de l'anglo-américain [...] aux yeux du public français qui associe d'une façon quasi automatique l'emploi de l'anglais [...] à l'idée de luxe, de qualité » (Etiemble, 1991 : 293). Le linguiste y refuse également le langage des médias qui s'efforcent de détruire la langue française en se référant à «un idiotisme américain ». Ce rejet fait un grand retentissement chez d'autres défenseurs de la langue: pour certains, « une insurmontable paresse, aggravée d'une coupable légèreté, serait cause de cette sorte de péché que constitue l'emprunt» (Treps, 2003 : 14), tandis que d'autres justifient son expansion par « le démon de la connaissance (exhibition des mots difficiles) et du snobisme qui s'y attache » (Rey-Debove, 1998 : 183). 
Dans une réaction conservatrice, apparaît l'adjectif «yankee » ${ }^{30}$ attribuant un sens péjoratif à tout ce qui est américain pendant que le terme anglicisme « est le plus souvent employé en introduction de glose, pour permettre au locuteur de prendre ses distances envers le mot anglais » (Steuckardt, 2006 : 10). Les autorités n'étant pas indifférentes envers cette question, prennent des mesures protectionnistes comme la promulgation de la loi Toubon en 1994 délimitant la place des emprunts en général et celle des anglicismes en particulier :

«Dans la désignation, l'offre, la présentation, le mode d'emploi ou d'utilisation, la description de l'étendue et des conditions de garantie d'un bien, d'un produit ou d'un service, ainsi que dans les factures et quittances, l'emploi de la langue française est obligatoire. Les mêmes dispositions s'appliquent à toute publicité écrite, parlée ou audiovisuelle » (Article 2 de la loi du 4 août $\left.1994^{31}\right)$.

Elles ont également mis en place des dispositifs d'enrichissement de la langue avec des néologismes. Elles ont créé des commissions terminologiques qui «ont pour mission de corriger ou prévenir l'anglicisation des vocabulaires techniques [...] en proposant diverses modalités de francisation »(Mortureux, 2001: 121). Elles ont rendu obligatoire l'emploi des termes publiés dans le Journal officiel de la République française.

Malgré ces tentatives de purisme, les cris des alarmistes pour sauvegarder l'autonomie socio-linguistique et donc politique du français passent pour vains. Ni la masse parlante ni les médias ne semblent obéir comme s'ils optaient pour une attitude de laxisme. Ils donnent feu vert aux emprunts pour s'implanter dans tous les domaines et sur tous les registres en dépit des équivalents français.

Cette attitude devient renforcée par l'esprit d'accueil et de tolérance adopté par l'autre camp des linguistes. Hagège s'est chargé de défendre ce phénomène linguistique en combattant les idées reçues sur ces prétendus dangers et surtout ceux de l'anglicisme :

\footnotetext{
${ }^{30}$ Adjectif relatif aux Américains des Etats-Unis.

${ }^{31}$ http://www.legifrance.gouv.fr/affichTexte.do?cidTexte=LEGITEXT000005616341 (consulté le 12 février 2015)
} 
«En dépit des prophéties souvent sombres qui s'entendent ici et là, rien n'indique que la langue française soit aujourd'hui submergée par un raz de marée d'anglicisme. Depuis, plus d'un millénaire, les usagers du français [...] traitent les emprunts en les digérant de mieux en mieux » (Hagège, 1987 : 71).

En effet, le français décèle une grande habileté à assimiler les emprunts venus des quatre coins du monde. Avec aisance, il s'approprie de tout ce qui lui est étranger à travers plusieurs stratégies d'intégration orthographique, sémantique, syntaxique et phonétique.

Cette attitude accueillante ne date pas d'aujourd'hui. Depuis le XII ${ }^{\text {ème }}$ siècle, la langue française s'est toujours montrée hospitalière vis-à-vis des nations qu'elle a côtoyées. Deroy avance cette même idée comme argument pour justifier le recours à l'emprunt. Selon lui, emprunter est incontournable puisque à chaque fois qu' "un groupe d'hommes parlant une langue définie se trouve en relations avec un autre groupe utilisant une langue distincte, il arrive presque toujours que des mots, des éléments grammaticaux, des significations s'introduisent d'un parler dans l'autre', (Deroy, $1980: 18$ ).

Ces mots voisins constituent un grand apport au français et contribuent à son renouvellement. D'une part, ils lui assurent une source inépuisable d'enrichissement lexical, d'autre part, ils représentent une immense occasion d'ouverture sur l'Autre. A ce titre, l'emprunt à l'anglais paraît inévitable et parfaitement légitime pour aller de pair avec les progrès scientifique et notamment technologique qui sont l'apanage des EtatsUnis. Contre le gré des puristes, le français, comme toutes les autres langues, insinue une foule de termes américains indispensables à une multitude de spécialisations telles que l'informatique, les sciences, la politique, le sport, le cinéma, les affaires et le marketing.

Loin de ces débats, les responsables du marketing et les créateurs des campagnes publicitaires n'accordent pas, à vrai dire, beaucoup d'attention ni aux alarmes des conservateurs ni aux propos des laxistes. Ils cherchent à tout prix à vendre, leur ultime objectif. Pour y arriver, ils ont découvert la meilleure solution : «voulez-vous vendre une robe ? yanquisez-la [...], 
accolez-lui l'adjectif le plus inattendu, le plus déplacé, celui, en effet, qu'on n'a jamais lu» (Etiemble, $1991: 296)$.

Ne prétendant point être les gardiens de la langue, les cyber-annonceurs trouvent dans l'anglicisme un moyen qui répond parfaitement à leurs besoins. Les règles de la mondialisation exigent que le commerce international fonctionne en anglais. Le dollar, étant la monnaie de référence mondiale, impose et la langue et le prestige de son pays. Le cybercommerce ne parle qu'en anglais. C'est également en anglais qu'est étiqueté un gigantesque nombre de produits. En outre, les concepteurs de publipostages sont conscients que l'usage de la langue maternelle est commun et n'a rien de spécial alors qu'ils visent à embobiner l'internaute, le dépayser et même le choquer. Pour présenter un discours insolite, ils substituent donc aux tournures habituelles d'autres importées d'outre-mer ou d'outre-Atlantique. Les raccourcis ainsi que la syntaxe claire et simple de cette langue correspondent aux besoins de brièveté et d'expressivité inédite. Un verbe anglais, une expression américaine pourraient rendre la phrase française plus originale, ce qui séduirait davantage les acheteurs. Considérons ces exemples :

(71). ' 'Une couleur pep's ${ }^{32}$ qui se marie à la perfection avec le motif graphique pour un look preppy ${ }^{33}$,' (Kiabi, 27/07/16)

(72). "Les pièces cool à adopter sans hésiter pour vos looks cet été !' (ASOS, 11/05/15)

(73). "Côté urbain : c'est le pull relief + la chemise denim qui lui feront accéder aux sphères urbaines du parfait gentleman!' (Jules, 02/10/13)

(74). "Les rayures ont soudain envie de singularité. Et les accessoires prennent leur rôle-trendy ${ }^{34}$ très à cœur" (Promod, 09/10/15)

(75). "Tee-shirt boyfriend femme! Voilà un tee-shirt qui s'adapte à toutes les situations et tous les styles !' (H\&M, $18 / 04 / 14)$

L'anglais jouit, en effet, d'un impact sans pareil, il « est bien souvent utilisé pour donner du lustre à ce qui n'en a pas, notamment dans le

\footnotetext{
${ }^{32}$ Pep : dynamisme et vivacité

${ }^{33}$ Preppy : ce qui a un style sobre et soigné, conforme à la tradition classique et bourgeoise.

${ }^{34}$ Trendy : qui est à la mode
} 
domaine du commerce, $[\ldots]$ pour ajouter une touche élégante » (Treps, 2003 : 242). Un produit qualifié de cool ou preppy donnant une allure de gentleman seraient mieux vendus. Un tee-shirt boyfriend ou un accessoire trendy qui ajoutent du pep's à votre tenue augmenteraient les chiffres d'affaires.

L'anglais révèle également une particularité souvent absente en français : l'art de la concision qui émane de la libre construction syntaxique et morphologique. Comparons ces deux exemples :

(76). "Nouvelle collection : directement dans votre wishlist!" (Mango, 11/12/15)

(77). "Retrouvez toutes nos idées cadeaux à offrir ou à glisser dans sa liste de væux de Noël." (Pimkie, 03/12/15)

La double synapsie « liste de vœux de Noël » est une unité complexe et assez longue. Elle devient facilement substituée par le terme anglais « wishlist » qui fournit le même signifié mais d'une façon plus concise. A travers cette comparaison, nous «évoquons la structure française qui exige la présence d'une préposition pour relier deux noms. [...]. Par contraste, l'anglais se passe entièrement de la préposition, de relation [...]. C'est une démarche [...] qui s'avère très efficace pour la création lexicale » (Picone, $1992: 11)$

N'oublions surtout pas que l'aspect caractéristique du cybermarketing est l'instantanéité. Pour attirer un internaute et le pousser à l'acte d'achat, le discours doit être rapide, concis et le plus souvent pas cher parce qu'avant tout, la publicité - pour le vendeur - n'est qu'un espace payant.

Par conséquent, à la place d'une longue périphrase en français, un seul terme anglais vient sauver la situation. Ainsi, il est plus pratique de dire :

(78). 'Ce brin sportwear inspire le confort et la chaleur' (Celio, $07 / 12 / 15)$

que : Ce brin vêtement de sport inspire le confort ..., sans oublier que le terme sport est lui-même un emprunt sans équivalent en français.

(79). “Découvrez les it de cet été'” (Camaïeu, 12/03/15) 
est beaucoup moins longue et donc plus efficace que : Découvrez les derniers produits de cet été, qu'il faut avoir pour être à la mode.

Parfois, le terme anglais attribue une connotation différente au signifiant français ou lui ajoute un nouveau sens.

(80). 'Laissez vos enfants se vêtir toute l'année à des prix discount'" (Tape à l'œil, 31/08/15)

(81). “Prêt à déclencher le french kiss ?"' (Jules, 19/12/14)

(82). "Découvrez notre sélection Hot de Noël” (Mango, 24/11/13)

Dans les exemples précédents, les emprunts ont été employés pour atténuer la connotation péjorative de prix (bas, réduits ou rabaissés) et du «baiser français ». De même, le choix de l'adjectif «hot» (ex. 82) manipule l'esprit des cyberclients : à l'opposé de l'équivalent français (chaud) renvoyant à la chaleur, cet adjectif revêt la sélection d'une attirance sexuelle.

Allant plus loin que le rôle passif d'un copieur, les concepteurs de publipostages français ne se contentent pas des emprunts intégraux et laissent libre cours à leur imagination favorisant ainsi la création des emprunts partiel, hybride ainsi que des faux-emprunts. Ils ne s'arrêtent pas - par exemple - à l'emploi de l'emprunt «shopping » mais ils en dérivent le verbe «shopper» dont l'usage est fréquent dans les publipostages ; puis le combinent récemment au morphème «e » (ex. 83). Ils dégagent de l'emprunt « fit » un adjectif « fittée » puis l'utilisent au superlatif (ex. 84).

(83). “J'e-shoppe mes shoes” (H\&M, 02/10/15)

(84). "Découvrez notre garde-robe revisitée en denim [...] Silhouette la plus fittée: Jean Ginger Fit 150€'” (Blancheporte, 24/05/16)

Une telle réaction créative est au cœur même de l'emprunt linguistique. Ce phénomène n'a jamais été un signe de paresse mais au contraire il témoigne d'une innovation au sein du système indigène, comme le montre Deroy :

« Le mot emprunté est en effet, par essence, un néologisme, c'est-à-dire une acception nouvelle introduite dans le 
vocabulaire d'une langue à une époque déterminée. Il se présente sous les différentes formes possibles $\mathrm{du}$ néologisme: mot nouveau, mot connu doté d'une signification nouvelle, mot transféré d'une catégorie grammaticale dans une autre $»($ Deroy, $1980: 11)$.

\section{Conclusion}

Malgré son penchant pour le purisme, la langue française ne s'est jamais enfermée dans son ghetto nationaliste. Elle s'est enrichie à l'aide d'une foule des mots débarqués de plusieurs langues. Parmi ces langues, l'anglais parait souverain: la majorité écrasante des emprunts dont regorge notre corpus s'avère être des anglicismes. Il ne se limite pas au plan morphologique, mais se manifeste également dans les autres composantes de la langue française (syntaxe, phonétique et sémantique). Si la pénurie lexicale rend certains emprunts nécessaires, d'autres dont le nombre est important semblent linguistiquement inutiles en présence de leurs équivalents français. Cependant, il faut se méfier de considérer ces emprunts comme des indices de l'effacement identitaire de la langue française. Au contraire, ces venants d'ailleurs ne font que manifester le génie du français capable de développer des stratégies d'absorption pour tout ce qui lui est étranger.

\section{Bibliographie}

- DEROY, Louis (1980), L'emprunt linguistique, Paris, Les Belles lettres.

- DUBOIS, Jean et al. (2002), Dictionnaire de linguistique et des sciences du langage, Paris, Larousse. 
- ETIEMBLE, René (1991), Parlez-vous franglais?, Paris, Gallimard, col. Folio/Actuel.

- GUILBERT, Louis (1975), La créativité lexicale, Paris, Larousse.

- HAGEGE, Claude (1987), Le français et les siècles, Paris, Odile Jacob.

- HUMBLEY, John (1974), «Vers une typologie de 1'emprunt linguistique », in Cahiers de lexicologie, vol. 2, no 25, (pp. 46-70), Paris, Didier/Larousse.

- LEHMANN, Alise et MARTIN-BERTHET, Françoise (2008), Introduction à la lexicologie sémantique et morphologique, Paris, Armand Colin.

- LEROY, Sarah (2006), «Glasnost et perestroïka. Les pérégrinations de deux russismes dans la presse française » in Mots. Les langages politiques, $\mathrm{n}^{\circ}$ 82 : L'emprunt et sa glose, article en ligne paru sur (consulté le 12 novembre 2014)

(2010), «Contacts et emprunts entre discours » in Congrès Mondial de Linguistique Française (CMLF 2010), Paris, Institut de Linguistique Française, article en ligne paru sur (consulté le 6 août 2016).

- MORTUREUX, Marie-Françoise (2001), La lexicologie entre langue et discours, Paris, Armand Colin, col. Campus Linguistique.

- PICONE, Michael D. (1992), «Le français face à l'anglais: aspects linguistiques » in Cahiers de l'Association internationale des études françaises, $\mathrm{n}^{\circ} 44, \quad$ (pp. 9-23), article en ligne paru sur http://www.persee.fr/doc/caief_0571-5865_1992_num_44_1_1775 (consulté le 20 mai 2015)

- REY-DEBOVE, Josette (1998), La linguistique du signe, Paris, Armand Colin, col. U, série Linguistique.

- STEUCKARDT, Agnès (2006), «L'anglicisme politique dans la seconde moitié du $18 \mathrm{e}$ siècle. De la glose d'accueil à l'occultation », in Mots, n'82/nov, (pp. 9-22), article en ligne paru sur http://mots.revues.org/746 (consulté le 01 octobre 2014)

- TREPS, Marie (2003), Les mots voyageurs, petite histoire du français venu d'ailleurs, Paris, Seuil.

- WALTER, Henriette (1997), L'aventure des mots français venus d'ailleurs, Paris, Robert Laffont.

- (2005), «L'intégration des mots venus d'ailleurs », in Alsic, Vol. 8, $\mathrm{n}^{\circ}$ 1, (pp. 35-44), article en ligne paru sur http://alsic.revues.org/324 (consulté le 07 novembre 2014).

\section{Abstract}

Parmi les voies exploitées quant à l'étude de la néologie, l'emprunt représente le mieux la voie la plus active vu le mouvement perpétuel au sein de son terrain 
d'activité. Un emprunt peut relever de différents ordres : orthographique, phonétique ou syntaxique et contribue par conséquent tant à l'enrichissement qu'à l'évolution du système linguistique accueillant. L'anglais - la langue du commerce mondial et du secteur numérique - s'avère être une source lexicale inépuisable pour les autres langues. Son intégration au sein du français dépend de plusieurs facteurs. Sur l'échelle de la nécessité, les anglicismes empruntés peuvent aller de l'indispensable jusqu'à l'inutile ; ce qui met en question l'apport néologique et créatif de ce phénomène dont l'emploi marque le discours du cybermarketing.

Mots clé : emprunt - anglicisme - discours - cybermarketing 


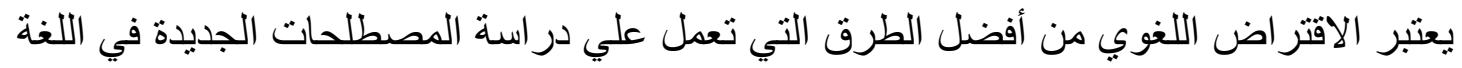

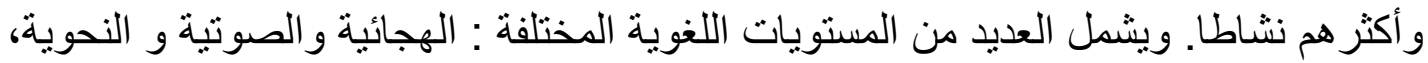

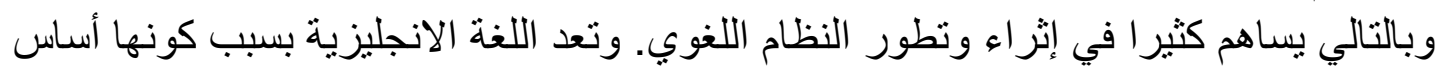

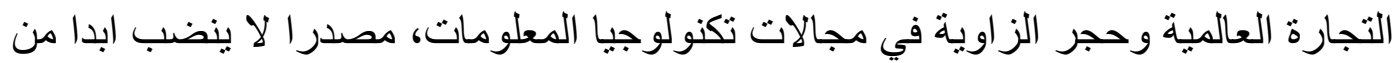

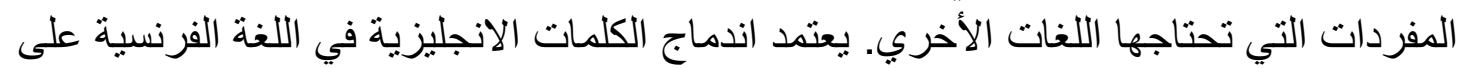

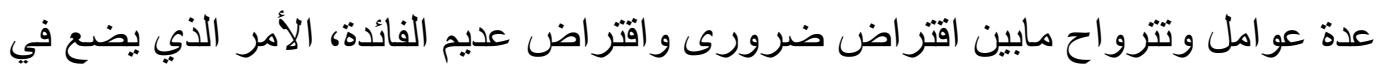
دائرة الثك المساهمة الإبداعية لهذه الظاهرة و التي تستخدم كثير ا في مجال التسويق الاكترونى.

$$
\text { الاكترونى المفتاحية : الاقتر اض اللغوى ـ المصطلحات الانجليزية ـ الخطاب ـ التنسويق }
$$

